\title{
ÁCAROS PARÁSITOS DE ROEDORES DE PLAYA DE ORO, COLIMA, MÉXICO
}

\author{
María Luisa Estébanes-González, ${ }^{1}$ Cornelio SÁnChez-Hernández, ${ }^{1}$ María \\ de Lourdes Romero-Almaraz ${ }^{2}$ y GARY D. Schnell ${ }^{3}$ \\ ${ }^{1}$ Departamento de Zoología, Instituto de Biología, Universidad Nacional Autónoma de México, \\ Apartado Postal 70-153, México, D. F. luisae@ibiologia.unam.mx, cornelio@servidor.unam.mx \\ ${ }^{2}$ Escuinapa 92 bis. Pedregal de Santo Domingo. Coyoacán, C. P. 04360. México, D. F. 1romero22@, \\ hotmail.com \\ ${ }^{3}$ Sam Noble Oklahoma Museum of Natural History and Department of Zoology, 2401 Chautauqua \\ Avenue, University of Oklahoma, Norman, OK 73072, USA. gschnell@ou.edu
}

Estébanes-González, M. L., C. Sánchez-Hernández, M. de L. Romero-Almaraz \& G. D. Schnell. 2011. Ácaros parásitos de roedores de Playa de Oro, Colima, México. Acta Zool. Mex. (n. s.), 27(1): $169-176$.

RESUMEN. Se reportan ácaros ectoparásitos en roedores colectados durante un estudio ecológico en Playa de Oro, en el estado de Colima, México. Los roedores revisados fueron: Heteromys pictus, Heteromys spectabilis, Baiomys musculus, Nyctomys sumichrasti, Oryzomys couesi, Oryzomys melanotis, Osgoodomys banderanus, Reithrodontomys fulvescens y Sigmodon mascotensis. Se registra la presencia de varias especies de ácaros de las familias Laelapidae, Macronyssidae, Listrophoridae y Myocoptidae en nuevos huéspedes y de algunas más se amplía su distribución. Se registra por primera vez para México a Lepidoglyphus hylandi, Laelaps oryzomydis y Prolistrophorus bakeri.

Palabras clave: Colima, Acari, Glycyphagidae, Laelapidae, Listrophoridae, Macronyssidae, Myocoptidae, Heteromyidae, Muridae.

Estébanes-González, M. L., C. Sánchez-Hernández, M. de L. Romero-Almaraz \& G. D. Schnell. 2011. Parasitic mites on rodents from Playa de Oro, Colima, Mexico. Acta Zool. Mex. (n. s.), 27(1): 169-176.

ABSTRACT. We report mites of rodents from Playa de Oro, Colima, Mexico. The genera of rodents sampled were: Heteromys pictus, Heteromys spectabilis, Baiomys musculus, Nyctomys sumichrasti, Oryzomys couesi, Oryzomys melanotis, Osgoodomys banderanus, Reithrodontomys fulvescens, and Sigmodon mascotensis. We recorded species of mites belonging to the families Laelapidae, Macronyssidae, Listrophoridae, and Myocoptidae that are on new hosts, whereas others are notable range extensions. Specimens collected represent first records from Mexico of Lepidoglyphus hylandi, Laelaps oryzomydis, and Prolistrophorus bakeri.

Key words: Colima, Acari, Glycyphagidae, Laelapidae, Listrophoridae, Macronyssidae, Myocoptidae, Heteromyidae, Muridae.

Recibido: 16/05/2008; aceptado: 08/01/2010. 


\section{INTRODUCCIÓN}

Los ácaros asociados a roedores se han estudiado de manera ocasional en México. Entre los primeros registros de parásitos de estos mamíferos en el occidente del país se encuentra el de Steptolaelaps liomydis colectado sobre Heteromys irroratus, en Jalisco (Grant 1947). De manera más constante a partir de la década de los 1970s, Anita Hoffmann e Isabel Bassols publicaron referencias de especies y localidades de ácaros parásitos (Hoffmann et al. 1972; Bassols 1975, 1981; Bassols et al. 1991, 1992). En general, los estudios han mostrado que dentro del orden Mesostigma la familia Laelapidae incluye a los principales parásitos de mamíferos pequeños (Estébanes \& Smiley 1997). Mientras que del orden Astigmata, la familia Listrophoridae se encuentra bien representada en la región Neotropical (Fain 1970, 1973), y los géneros mejor conocidos en México son Prolistrophorus (Fain 1970, 1973) y Geomylichus (Servin et al. 1992, 1994; Vargas et al. 1999; Fain \& Estébanes 2000).

Debido a que los estudios sobre ácaros ectoparásitos (hematófagos) son limitados, y particularmente los del occidente de México, es de interés presentar información al respecto, para Playa de Oro, Colima, México.

\section{MATERIAL Y MÉTODOS}

Área de estudio. Playa de Oro, Colima, se encuentra a $10 \mathrm{~m}$ snm, entre las coordenadas: $19^{\circ} 08.057^{\prime} \mathrm{N}$ y $104^{\circ} 29.969^{\prime} \mathrm{O}$. La vegetación dominante es bosque tropical caducifolio, con bosque espinoso y algunos manchones de mangle. Algunos árboles y arbustos dominantes son Coccoloba barbadensis (Juan Pérez), varias especies de Acacia como A. hindis (bull horn Acacia) y A. farnesiana (huizache), Senna pallida (biche silvestre) y $S$. occidentalis (biche prieto), Pithecellobium lanceolatum y $P$. dulce (guamúchil), Hyperbaena ilicifolia, Crataeva tapia (barna, varum), Prosopis juliflora (mezquite común) y Guazuma ulmifolia (guácimo); además de que una parte importante del área se destina a la agricultura y al cultivo de palma de coco (Cocos nucifera; Schnell et al. 2008).

Trabajo de campo. La recolecta de los ácaros se realizó del 1 al 13 de enero del 2003. Para la captura de los roedores se utilizaron trampas de aluminio, plegadizas, tipo Sherman, que fueron cebadas con hojuelas de avena, y se colocaron en cinco cuadrantes de una hectárea cada uno, formados por diez líneas con diez estaciones de captura, a una distancia de $10 \mathrm{~m}$ entre cada trampa. Debido a que uno de los objetivos de este proyecto fue la captura y recaptura de los roedores, sólo se conservaron y examinaron aquellos ejemplares que murieron por estrés y algunos de los que se capturaron el último día de trabajo en cada cuadrante, para corroborar la identificación de las especies. En total se revisaron 84 especímenes que se cepillaron en sentido contrario al nacimiento del pelo con la finalidad de obtener los ácaros asociados a ellos. 
Los roedores recolectados forman parte del proyecto "Mamíferos de Colima" que se realiza por parte de la Universidad de Oklahoma, la Universidad de Memphis, la Universidad de Auburn y la Universidad Nacional Autónoma de México. Actualmente se encuentran en proceso de estudio, pero serán depositados en la Colección Nacional de Mamíferos (Instituto de Biología, UNAM) y la Colección de Mamíferos del Sam Noble Museum of Natural History, de la Universidad de Oklahoma.

Los ácaros se conservaron en tubos viales con alcohol al $70^{\circ}$ y fueron etiquetados con el número de colecta del huésped. Una vez en el laboratorio se montaron en preparaciones fijas en líquido de Hoyer. El material montado, etiquetado y clasificado se encuentra en la Colección Nacional de Ácaros del Instituto de Biología de la Universidad Nacional Autónoma de México y en la colección del autor principal.

\section{RESULTADOS}

Los 84 ejemplares de roedores se agrupan en 9 especies, 2 de la familia Heteromyidae: Heteromys pictus $(9 ; 10.71 \%)$ y Heteromys spectabilis $(5,5.95 \%)$; y 7 de la familia Muridae: Baiomys musculus (14; 16.67\%), Nyctomys sumichrasti $(3,3.57 \%)$, Oryzomys couesi (42, 50.0\%), Oryzomys melanotis (1, 1.19\%), Osgoodomys banderanus (1, 1.19\%), Reithrodontomys fulvescens (4, 4.76\%) y Sigmodon mascotensis (5, 5.95\%) (Fig. 1). Por su parte, los ácaros se agrupan en 13 especies de los órdenes Mesostigmata y Astigmata. El primero incluye a las familias Laelapidae y Macronyssidae; y el segundo está representado por Acaridae, Glycyphagidae, Listrophoridae y Myocoptidae.

Los ácaros de la familia Listrophoridae se encontraron sobre las nueve especies de roedores examinadas, mientras que los de la familia Laelapidae faltaron únicamente en Sigmodon mascotensis. Los ácaros que se encontraron en un mayor número de huéspedes fueron Prolistrophorus frontalis (Baiomys musculus, Nyctomys sumichrasti, Oryzomys couesi, Oryzomys melanotis y Osgoodomys banderanus) y Echinonyssus microchelae (Heteromys pictus, Heteromys spectabilis, Oryzomys couesi y Reithrodontomys fulvescens). Cinco especies de ácaros se encontraron sólo en una especie de roedor: Androlaelaps spinosus sobre Osgoodomys banderanus; Ornithonyssus bacoti sobre Oryzomys couesi; Tyrophagus putrescentiae y Lepidoglyphus hylandi sobre Baiomys musculus; y Myocoptes musculinus sobre Reithrodontomys fulvescens. El roedor con el mayor número de parásitos fue Oryzomys couesi con cinco especies (Laelaps oryzomydis, Echinonyssus microchelae, Ornithonyssus bacoti, Prolistrophorus frontalis y Prolistrophorus bakeri), seguida de Baiomys musculus (Androlaelaps fahrenholzi, Tyrophagus putrescentiae, Lepidoglyphus hylandi y Prolistrophorus frontalis); mientras que sobre Sigmodon mascotensis sólo se encontró a Prolistrophorus bakeri (cuadro 1; Fig. 1). 


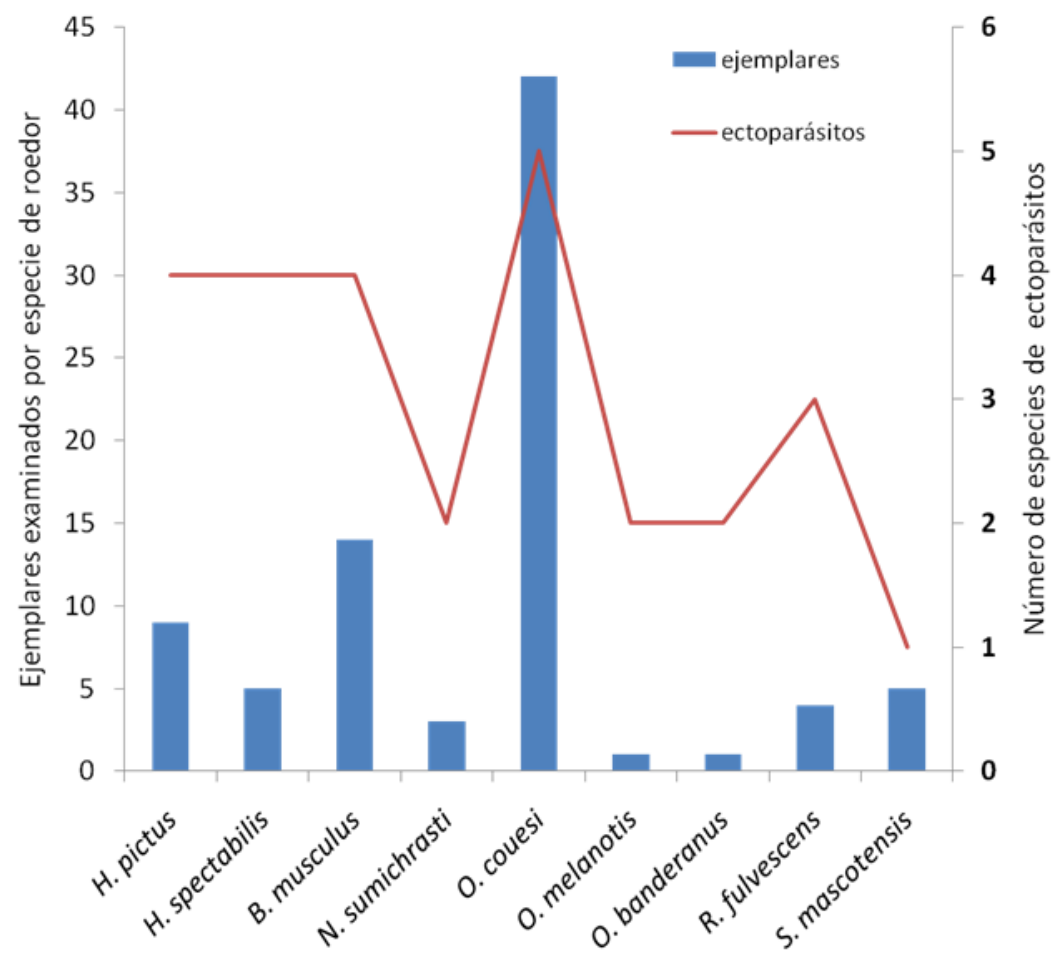

Fig. 1. Número de especímenes examinados de cada especie de roedor y número de especies de ácaros asociadas.

\section{DISCUSIÓN}

Tres especies de ácaros se citan por primera vez para México (Laelaps oryzomydis, Lepidoglyphus hylandi y Prolistrophorus bakeri) y siete por primera vez para Colima (Androlaelaps casalis, Androlaelaps fahrenholzi, Androlaelaps spinosus, Echinonyssus microchelae, Ornithonyssus bacoti, Prolistrophorus frontalis y Myocoptes musculinus). Mientras que seis especies de ácaros se citan sobre huéspedes nuevos: Androlaelaps casalis sobre Heteromys spectabilis; Androlaelaps fahrenholzi sobre Baiomys musculus y Nyctomys sumichrasti; Lepidoglyphus hylandi sobre Baiomys musculus; Geomylichus postscutatus sobre Heteromys pictus y Heteromys spectabilis; Prolistrophorus frontalis sobre Baiomys musculus, Nyctomys sumichrasti, Oryzomys couesi, Oryzomys melanotis y Osgoodomys banderanus; y Myocoptes musculinus sobre Reitrodontomys fulvescens (Cuadro 1).

La familia de ácaros ectoparásitos mejor representada en los roedores de Playa de Oro, Colima, fue Laelapidae con 6 especies, de las cuales Steptolaelaps liomydis es la de mayor distribución en México. Sus huéspedes pertenecen al género Heteromys, 
Acta Zool. Mex. (n.s.) 27(1) (2011)

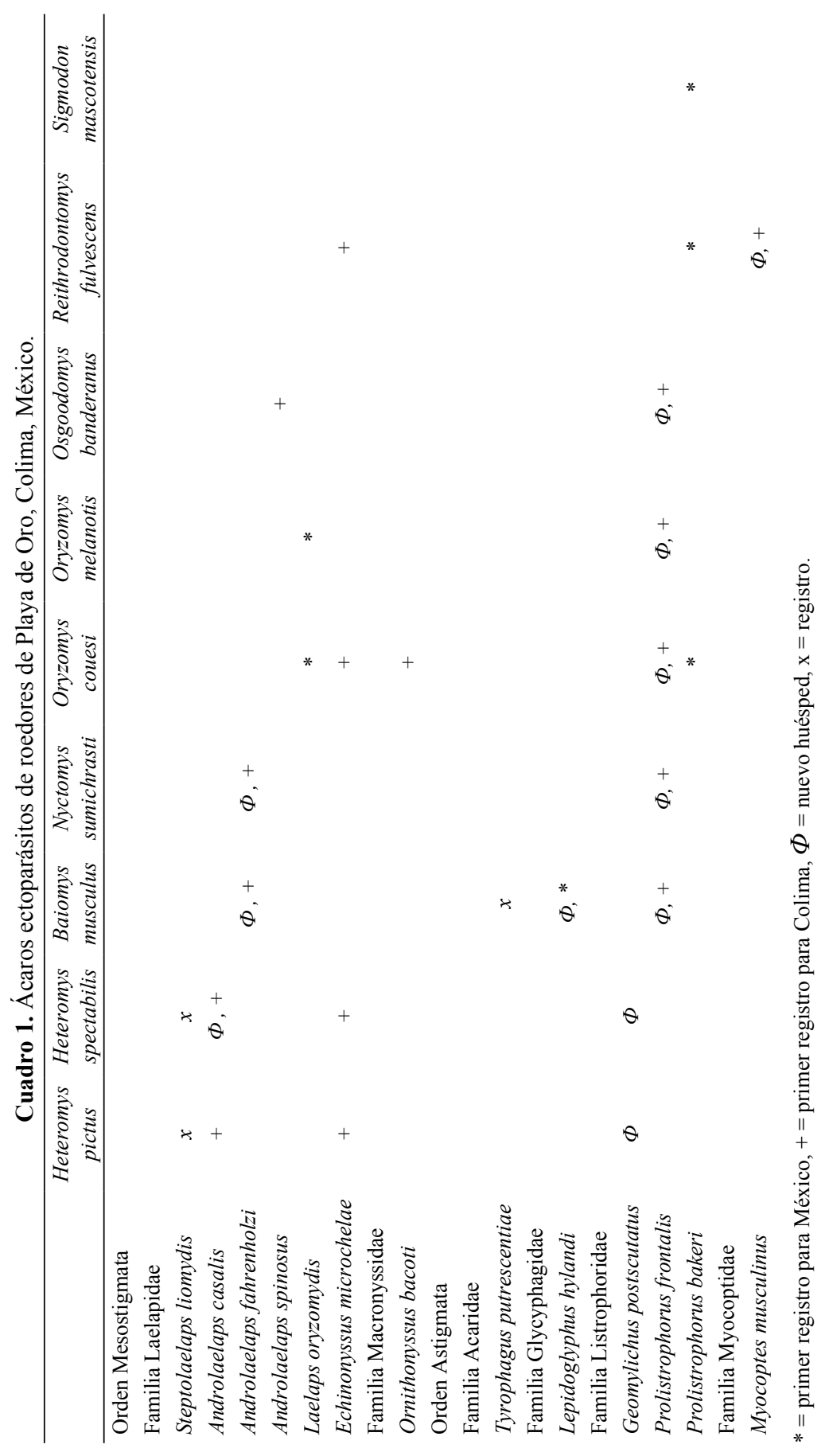


aunque también se han recolectado sobre Perognathus spinatus, Oligoryzomys fulvescens, Peromyscus truei, Reithrodontomys sp. (Bassols 1975) y Sigmodon hispidus (Estébanes-González \& Cervantes 2005). Androlaelaps casalis se citó por primera vez en México en el estado de Nayarit sobre Heteromys pictus y en el Estado de México sobre Heteromys sp. (Bassols 1975). Al parecer son parásitos frecuentes de ardillas (Strandtmann 1949), pero se encuentra en una gran variedad de huéspedes y se distribuye en casi todo el mundo. Androlaelaps fahrenholzi tiene una distribución cosmopolita, en México se ha reportado en 14 estados y en 40 especies de huéspedes (Bassols 1981, Vargas-Sandoval 1991, Quintero et al. 2001, Estébanes-González \& Cervantes 2005, Whitaker, Jr. \& Morales-Malacara 2005); mientras que Androlaelaps spinosus sólo se había reportado para Guerrero sobre Osgoodomys banderanus y Peromyscus sp. (Bassols 1975) y en Michoacán sobre Peromyscus sp. (Furman 1955). Por su parte, la única especie de la familia Macronyssidae, Ornithonyssus bacoti, tiene una distribución cosmopolita, aunque se cree que se originó en el nuevo mundo como parásito de Sigmodon hispidus (Strandmann \& Wharton 1958).

El Orden Astigmata incluye a la familia Acaridae y la especie Tyrophagus putrescentiae, la cual es micófaga (Hughes 1979) y llega de forma incidental a los roedores. Los representantes de las familias Glycyphagidae y Listrophoridae se asocian al pelo de los mamíferos; la primera incluye a Lepidoglyphus hylandi; esta especie se colectó en forma de hipopodio pilicola, se sujeta por medio de agarraderas ventrales y daña la base de los folículos pilosos. El hipopodio es el único estado conocido de la mayoría de las especies de esta familia de glicifágidos, por lo que se desconoce información sobre su biología; aunque en algunas especies se ha estudiado su ciclo de vida y entre éstas se encuentra Lepidoglyphus hylandi (Fain \& Spicka 1986).

La familia Listrophoridae es muy diversa y las poblaciones de sus especies son numerosas; de éstas, Geomylichus postscutatus es el segundo registro para Colima, y en México se había recolectado sobre Heteromys irroratus en San Luis Potosí, en El Cóbano, Colima (Fain et al. 1978), en Jalisco y Nuevo León (Vargas et al. 1999). Prolistrophorus frontalis se recolectó sobre cinco huéspedes nuevos; anteriormente sólo se había encontrado sobre Oryzomys fulvescens en el estado de Veracruz (Estébanes-González \& Cervantes 2005). Este género está confinado a la región neotropical del continente americano sobre roedores de la familia Cricetidae (Fain \& Estébanes 1996). Myocoptes musculinus perteneciente a la familia Myocoptidae, produce daños considerables sobre los roedores porque ataca el pelo de la cara y de otras partes del cuerpo (Watson 1960).

AGRADECIMIENTOS. Expresamos nuestro reconocimiento a los Dres. M. L. Kennedy, T. L. Best, R. D. Owen y C. Dick, así como a numerosos estudiantes de México y los Estados Unidos de Norteamérica, quienes participaron activamente en el campo. El permiso de colector fue otorgado a C. Sánchez-Hernández, por el Instituto Nacional de Ecología, Dirección General de Vida Silvestre clave FAUT.0103. 


\section{LITERATURA CITADA}

Bassols, B.I. 1975. Mesostigmata de los mamiferos de México, con especial referencia a la subfamilia Laelapinae (Acari: Parasitiformes). Tesis de Doctorado de la Escuela Nacional de Ciencias Biológicas, Instituto Politécnico Nacional. México, D.F.

Bassols, B.I. 1981. Catálogo de los ácaros Mesostigmata de mamíferos de México. Anales de la Escuela Nacional de Ciencias Biológicas. 24:9-49.

Bassols-Batalla, I., M.T. Quintero Martínez, J.A. Moreno-Moreno \& T. Vessi-Lobato. 1991. Ácaros del género Hirstionyssus Fonseca en México, con descripción de una nueva especie (Mesostigmata, Laelapidae). Anales de la Escuela Nacional de Ciencias Biológicas. 34:145-157.

Bassols-B., I., M. Vargas \& O.J. Polaco. 1992. El género Hirstionyssus Fonseca, 1948 (Laelapidae: Hirstionyssinae) en México. Memoria del Ciclo de Conferencias Parasicológicas in memorian del Dr. Antonio Acevedo Hernández. Facultad de Medicina Veterinaria y Zootecnia, Universidad Nacional Autónoma de México, México, D. F.

Estébanes-González, M.L. \& F.A. Cervantes. 2005. Mites and ticks associated with some small mammals in Mexico. International Journal of Acarology. 31:23-37.

Estébanes-González, M.L. \& R.L. Smiley. 1997. Two new species of the genus Echinonyssus Hirst (Acari: Laelapidae) from Mexico, with a key to the Neotropical species. International Journal of Acarology. 23:237-242.

Fain, A. 1970. Diagnoses de nouveaux Lobalgides et Listrophorides (Acarina: Sarcoptiformes). Revue de Zoologie et de Botanique Africaines. 81:271-300.

Fain, A. 1973. Les Listrophoridés d'Amérique Néotropicale (Acarina: Sarcoptiformes). I. Familles Listrophoridae et Chirodiscidae. Bulletin de l'Institut Royal des Sciences Naturelles de Belgique. 49:1-149.

Fain, A. \& M.L. Estébanes. 1996. New fur mites of the family Listrophoridae (Acari: Astigmata) from Mexico. International Journal of Acarology. 22:181-186.

Fain, A. \& M.L. Estébanes. 2000. Notes on the fur mites of the genus Geomylichus Fain, 1970 (Acari, Astigmata, Listrophoridae), with description of a new species from Mexico. Bulletin et Annales de la Société Royale Belge d'Entomologie. 136:53-59.

Fain, A. \& E.J. Spicka. 1986. The life-cycle of Lepidoglyphus hylandi (Fain, 1969) comb. nov. (Acari, Glycyphagidae). Acarologia. 27:317-324.

Fain, A., J.O. Whitaker Jr., T.G. Schwan \& F.S. Lukoschus. 1978. Notes on the genus Geomylichus Fain, 1970 (Astigmata: Listrophoridae) and description of six new species. International Journal of Acarology. 4:101-114.

Furman, D.P. 1955. Steptolaelaps (Acarina: Laelapidae) a new genus of the mites parasitic on neotropical rodents. Journal of Parasitology. 41:519-525.

Grant, D.C. 1947. North American mites of the genus Laelaps (Arachnida:Acarina: Parasitidae). Microentomology. 12:1-21.

Hoffmann, A., I.B. de Barrera \& C. Méndez. 1972. Nuevos hallazgos de ácaros en México. Revista de la Sociedad Mexicana de Historia Natural. 33:151-159.

Hughes, A.M. 1979. The mites of stored food and houses. Techical Bulletin 9. Her Majesty's Stationery Office, London.

Quintero, M.M.T., M. Vargas, B.S. Hernández, P. García \& N.J. Otero. 2001. Ectoparasitic mites on Heteromys gaumeri in the South of Yucatan, Mexico. Pp. 583-585. In: R.B. Halliday, D.E. Walter, H.C. Proctor, R.A. Norton and M.J. Colloff(Eds). Acarology: Proceeding of the 10th International Congress. CSIRO Publishing, Melburne, Australia.

Schnell, G.D., M.L. Kennedy, C. Sánchez-Hernández, M.L. Romero-Almaraz, B.D.N. Estevez, J.A. Guerrero, T.L. Best, M.C. Wooten \& R.D. Owen. 2008. Habitat preference of the endemic 
tawny deermouse (Peromyscus perfulvus), a species of conservation concern. Southwestern Naturalist. 53:9-20.

Servin, R., R. Aguilar \& S.T. Álvarez-Castañeda. 1992. Una nueva especie del género Geomylichus Fain, 1970, de isla Cerralvo: Baja California Sur, México. Southwestern Entomologist. 17:341345.

Servin, R., R. Aguilar \& S.T. Álvarez-Castañeda. 1994. Una nueva especie del género Geomylichus Fain, 1970, presente en un roedor endémico de Baja California Sur, México. Southwestern Entomologist. 19:285-289.

Strandtmann, R.W. 1949. The blood-sucking mites of the genus Haemolaelaps (Acarina: Laelaptidae) in the United States. Journal of Parasitology. 35:325-352.

Strandmann, R.W. \& G.W. Wharton. 1958. A manual of Mesostigmatid mites parasitic on vertebrates. Institute of Acarology, Maryland, U. S. A. Contribution 4:1-330.

Vargas-Sandoval, M. 1991. Los artrópodos asociados a los mamíferos de la Reserva de la Biosfera de Mapimí, Durango, con especial atención a los ácaros Mesostigmados. Tesis de Licenciatura. Instituto Politécnico Nacional, Escuela Nacional de Ciencias Biológicas, México, D.F.

Vargas, M., T.M. Pérez \& O.J. Polaco. 1999. The genus Geomylichus Fain (Acari: Listrophoridae) from Mexico, with descriptions of four new species. International Journal of Acarology. 25:3-12.

Watson, D.P. 1960. On the adult and immature stages of Myocoptes musculinus (Koch) with notes on its biology and classification. Acarologia. 2:335-344.

Whitaker, Jr. J. O. \& J. B. Morales-Malacara. 2005. Ectoparasites and other associates (Ectodytes) of Mammals of Mexico, pp. 535-666. In: V. Sánchez-Cordero, R. A. Medellín (Eds). Contribuciones Mastozoológicas en Homenaje a Bernardo Villa. Instituto de Biología, UNAM; Instituto de Ecología, UNAM; CONABIO, México. 